\title{
Increased spontaneous recombination in RNase H2- deficient cells arises from multiple contiguous rNMPs and not from single rNMP residues incorporated by DNA polymerase epsilon
}

\author{
Anastasiya Epshtein ${ }^{1}$, Catherine J. Potenski ${ }^{2}$, and Hannah L. Klein ${ }^{1, *}$ \\ ${ }^{1}$ Department of Biochemistry and Molecular Pharmacology, New York University School of Medicine, 550 First Avenue, New York, \\ New York 10016, USA. \\ ${ }^{2}$ Nature Publishing Group, One New York Plaza, New York, New York 10004, USA. \\ * Corresponding Author: \\ Hannah L. Klein, Department of Biochemistry and Molecular Pharmacology, Smilow 201C, 522 First Avenue, NYU Langone Medical \\ Center; New York, NY 10016, USA; Tel: +1 212263 9484, Fax: +1 212263 9027; E-mail: hannah.klein@nyumc.org
}

\begin{abstract}
Ribonucleotides can become embedded in DNA from insertion by DNA polymerases, failure to remove Okazaki fragment primers, R-loops that can prime replication, and RNA/CDNA-mediated recombination. RNA:DNA hybrids are removed by RNase $H$ enzymes. Single rNMPs in DNA are removed by RNase $\mathrm{H} 2$ and if they remain on the leading strand, can lead to mutagenesis in a Top1-dependent pathway. rNMPs in DNA can also stimulate genome instability, among which are homologous recombination gene conversion events. We previously found that, similar to the rNMP-stimulated mutagenesis, rNMP-stimulated recombination was also Top1-dependent. However, in contrast to mutagenesis, we report here that recombination is not stimulated by rNMPs incorporated by the replicative polymerase epsilon. Instead, recombination seems to be stimulated by multiple contiguous rNMPs, which may arise from R-loops or replication priming events.
\end{abstract}

\author{
doi: 10.15698/mic2016.06.506 \\ Received originally: 01.03.2016; \\ in revised form: 04.05.2016, \\ Accepted 09.05.2016, \\ Published 15.05.2016.
}

Keywords: RNase $\mathrm{H} 2$, recombination, rNMP, DNA polymerase.

\section{Abbreviations:}

WT - wild type.

\section{INTRODUCTION}

In the last few years it has become apparent that the replicative polymerases misincorporate rNMP residues into DNA at rates that may become a significant burden to genome stability. It has been estimated that during replication about 15,000 rNMP become incorporated into the nuclear genome of the yeast Saccharomyces cerevisiae [1]. These rNMP residues can distort the DNA helix and impede progression of RNA and DNA polymerase machineries. Additional sources of rNMPs in DNA may come from incomplete removal of the Okazaki fragment primers. The embedded rNMPs are removed quite efficiently by the nuclease RNase $\mathrm{H} 2$, which recognizes both single and multiple rNMP residues in a RNA:DNA hybrid conformation [2, 3] or by RNase $\mathrm{H} 1$, which has specificity towards multiple contiguous rNMP residues of four or more paired with dNMPs [2]. The process of removal of single rNMP residues during replication is termed ribonucleotide excision repair (RER) [4].

The RNase $\mathrm{H} 1$ ribonuclease acts primarily on R-loops, which differ in structure from rNMPs embedded in DNA. R- loops are formed during replication and have a RNA:DNA duplex strand opposite an unpaired single DNA strand. Rloops also form during high transcription [5-8], often the result of transcription replication collisions $[6,9,10]$. Detection using an antibody against RNA:DNA hybrids has revealed the natural occurrence of these hybrids at highly transcribed genes, tRNA genes, Ty sequences, and telomeres $[11,12]$.

High transcription, R-loop formation and transcription replication collisions are known to stimulate DNA break formation and recombination [5, 8, 13-15]. High transcription can result in increased gene conversion [15], but it is not known whether this is associated with R-loop formation [13]. R-loop formation results in increased recombination that can be reduced by over-expression of RNase $H 1[6,9,16]$, suggesting that persistent R-loops can provoke recombination-promoting lesions. Further evidence for a role for transcription and RNase $\mathrm{H}$ comes from studies on gross chromosomal rearrangements, which are reduced when RNase $\mathrm{H} 1$ is overexpressed in cells with altered transcription and chromatin structure [14]. Recently, transcrip- 
tion-dependent R-loops have been shown to be able to initiate replication at regions other than replication origins and cause genome instability from endoreduplication and copy number changes [17].

In the absence of functional RNase $\mathrm{H} 2$ enzyme, rNMPs remain in DNA, as evidenced by the alkali sensitivity of genomic DNA [18]. rNMPs on the leading strand can be cleaved by the action of Topoisomerase I (Top1) [19, 20], generating a nick that can lead to further processing and signature deletion mutations in simple repeats $[19,21]$.

In addition to deletion mutations, loss of RNase $\mathrm{H} 2$ function is characterized by hyper-recombination between directly repeated sequences [22], chromosome loss [23], and chromosome rearrangements [23]. Indeed, a mutation in an RNase $\mathrm{H} 2$ subunit was recovered as a hyper-rec mu- tant in an early screen for such mutants [24]. Studies on the susceptibility of hyper-recombination to Top 1 action in an RNase H2-defective cell environment showed that similar to increased deletion mutation, hyper-recombination also sensitive to Top1 and is not stimulated in a Top1defective strain. This finding promoted us to further explore the nature of the initiating lesion for hyperrecombination in the absence of RNase $\mathrm{H} 2$.

\section{RESULTS}

RNase $\mathrm{H} 2$ mutants have an increased recombination phenotype

In our collection of hyper-rec (hpr) mutants that we isolated on the basis of increased intrachromosomal recombination [24] was the hpr4-1 mutant. We were intrigued by this
A

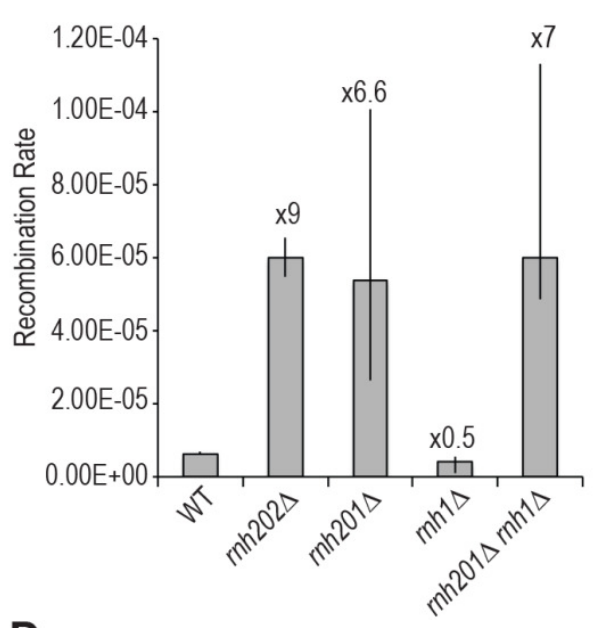

D

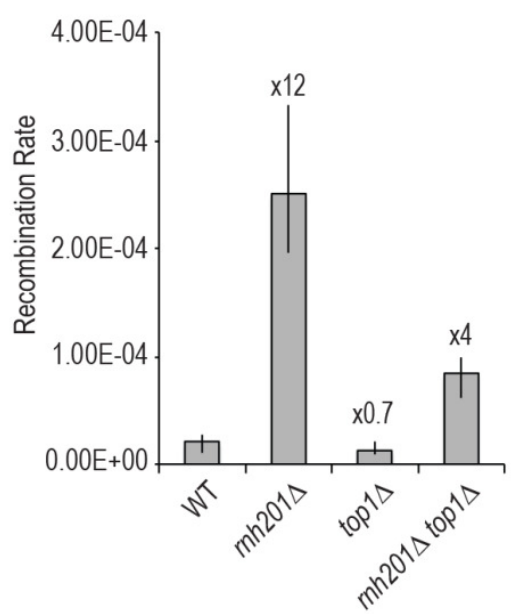

B

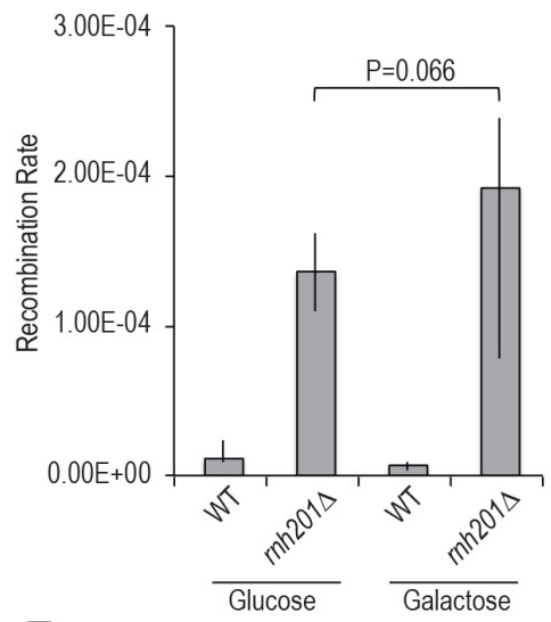

E

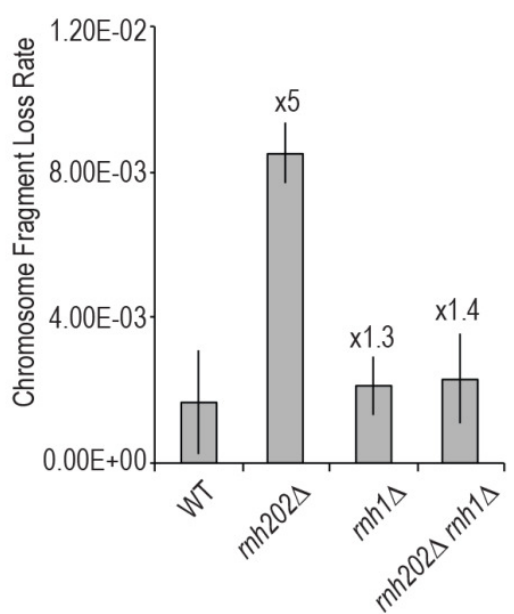

C

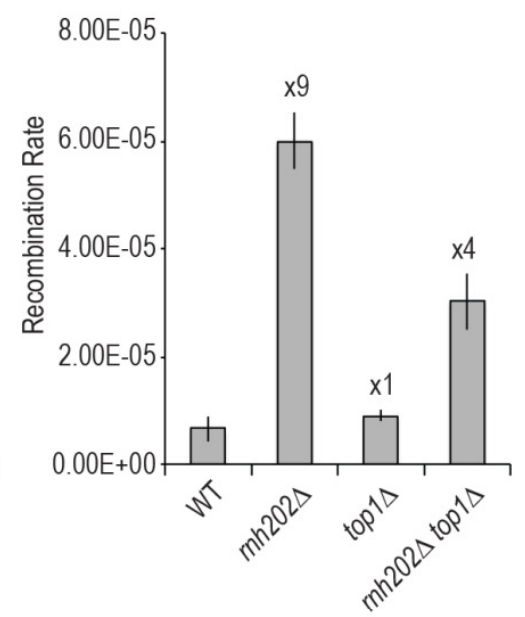

FIGURE 1: Intrachromosomal gene conversion is increased in RNase H2-defective cells. (A) Recombination rates of gene conversion are shown as median rates with $95 \%$ confidence limits $(n=18)$ in RNase $\mathrm{H} 1$ and RNase $\mathrm{H} 2$ mutant strains. The reporter is a duplication of leu2 genes at the LEU2 locus. (B) Effect of RNase $\mathrm{H} 1$ over-expression in intrachoromosomal gene conversion rates. Recombination rates of the ade2 duplication reporter with a Gal-Rnh1 plasmid are shown with 95\% confidence limits $(n=12)$. (C) Effect of TOP1 deletion on the gene conversion recombination rate using the leu2 duplication reporter. The wild type (WT) and $r n h 202 \Delta$ rates are taken from panel A. (D) Recombination rates of gene conversion are shown as median rates with $95 \%$ confidence limits $(n=18)$ in RNase $\mathrm{H} 2$ mutant strains using a different recombination reporter consisting of a duplication of ade2 genes at the ADE2 locus. The effect of TOP1 deletion on this reporter is also shown. (E) Chromosome fragment loss in a haploid strain is shown. Median rates with standard deviations are displayed. 
mutant as it showed an increased rate of mitotic gene conversion, but did not deviate in the pattern of recombination events from wild type. Moreover, the mutant also had a mutator phenotype while displaying no DNA damage sensitivity, but was synthetically sick with mutants of the MRX complex. This suggested a defect in processing DNA break damage or a bypass pathway that could not process all DNA break damage. Subsequent studies identified hpr41 as an allele of RNH2O2, a subunit of yeast RNase H2 [22].

To further characterize the hyper-rec phenotype, we determined recombination rates in an $r n h 202 \Delta$ strain, in addition to rates from an $r n h 201 \Delta$ strain and an $r n h 1 \Delta$ strain. While the rhn201 and rnh202 mutants had similar elevated recombination rates, recombination in the $r n h 1$ strain did not change from wild type (Figure 1A). Moreover, addition of the rnh1 mutation to an rnh202 strain did not enhance the rnh202 recombination rate (Figure 1A). Overexpression of RNase $\mathrm{H} 1$ did not reduce the increased recombination seen in an rnh201 mutant (Figure 1B), further suggesting that the increased gene conversion does not arise from a substrate that accumulates in the absence of RNase $\mathrm{H} 1$.

Recent reports have found that using a global genome analysis of RNase $\mathrm{H} 2$-defective strains there is a widespread occurrence of recombination and loss of heterozygosity in diploid strains $[25,26]$. As the increased mutation rates observed in $r n h 2$ mutants are dependent on Top1 [18, $19,21,22]$, and one report found that loss of heterozygosity crossing over and nonallelic recombination was Top1dependent [25], we examined recombination in an

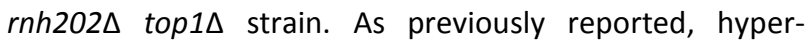
recombination in $r n h 202 \Delta$ requires Top1 activity [22] (Figure 1C) and we observed a similar Top1-dependence using the second recombination reporter (Figure 1D). We used two different recombination reporters because we wanted to verify that the increased recombination, gene conversion, was not reporter specific or genome location specific. The genome-wide studies of recombination increase have focused on loss of heterozygosity and reciprocal crossing over in diploids $[25,26]$ whereas in this case we have examined gene conversion in haploids. In all cases, loss of RNase $\mathrm{H} 2$ has resulted in increased recombination and genome instability.

\section{Increased genome instability is observed in RNase H2- defective strains}

Our early studies revealed that the rnh202A mutant has additional genome instability phenotypes of chromosome loss that are also observed in a mouse RNase $\mathrm{H} 2$ knockout line [23] as well as in genome studies of yeast RNase H2defective cells $[25,26]$. To further measure spontaneous genome instability, we determined loss of a chromosome fragment in haploid rnh202A strains (Figure 1E). Loss of RNase $\mathrm{H} 2$ function greatly stimulated genome instability, while loss of RNase $\mathrm{H} 1$ did not result in an increase. Curiously in the double RNase $\mathrm{H}$ mutant the $r n h 1 \Delta$ rate was observed, suggesting that processing of rnh2-lesions by $\mathrm{RNase} \mathrm{H} 1$ leads to chromosome instability.

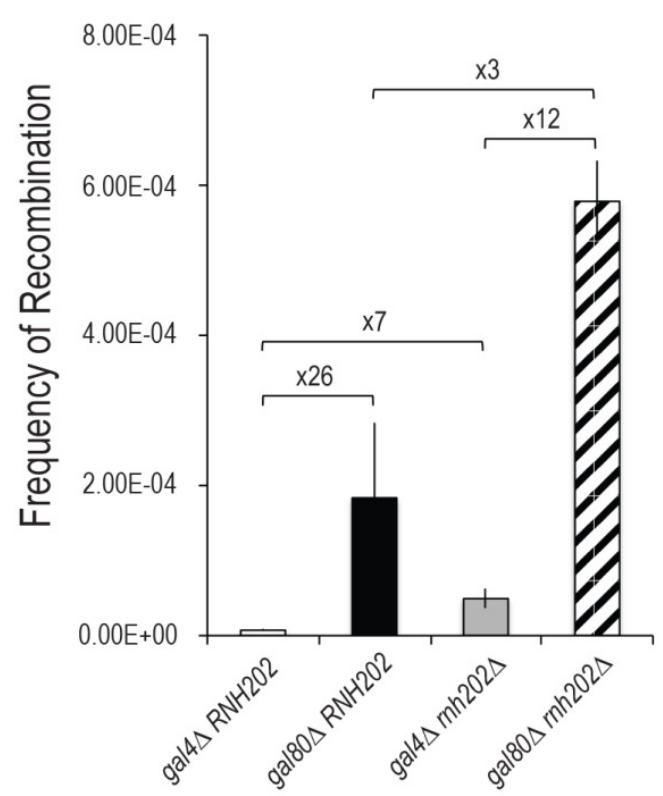

FIGURE 2: Transcription stimulation of recombination is independent of $r n h 202$ stimulation of recombination. Recombination between duplicated gal10 genes at the GAL10 locus as a function of transcription status is shown as median rates with standard deviations ( $n=27$ ). Low transcription occurs in gal4 $\Delta$ strains while high transcription occurs in gal80 $\Delta$ strains. Fold differences between indicated genotypes are shown.

\section{Increased recombination is stimulated by high transcrip- tion}

Recombination can be stimulated by high transcription [13, 15]. To determine if recombination in an $r n h 202 \Delta$ strain was linked to transcription, we examined recombination in a reporter under low and high transcription conditions. The reporter was a duplication of GAL10 genes, each with a different mutation, in strains with low transcription (ga/4) and high transcription (ga/80) levels of the GAL10 genes (Figure 2). In a wild type strain, recombination was stimulated 26-fold, as previously reported [15]. Similarly, in an rnh202A strain recombination was stimulated under high transcription conditions. The increase was more than additive, suggesting that in a recombination permissive state, that is, under high transcription, loss of RNase $\mathrm{H} 2$ increases transcription-stimulated recombination.

Increased rNMP incorporation into DNA does not alter recombination rates

rNMP residues can become embedded in DNA through the action of the replicative polymerases. These can stimulate mutation as use of a DNA polymerase epsilon allele, pol2M644G, that has reduced sugar specificity and allows 10fold higher incorporation of rNMPs into DNA, also increases deletion mutations [18], dependent on Top1 activity [19, $21,27]$. Similarly, the pol2-M644L allele, which reduces rNMP incorporation, also reduces the rate of deletion mutations. As we have found that the rnh2024 hyperrecombination is dependent on Top1 function, we determined recombination rates in rnh2024 pol2-M644G and 
rnh202A pol2-M644L strains (Figure 3A-B). Unexpectedly, we found that the polymerase epsilon allele pol2-M644G did not increase the recombination rates, as would be expected, and the polymerase epsilon allele po/2-M644L did not reduce the rate using the leu2 duplication reporter and only slightly reduced the rate with the ade2 duplication reporter.

\section{A}

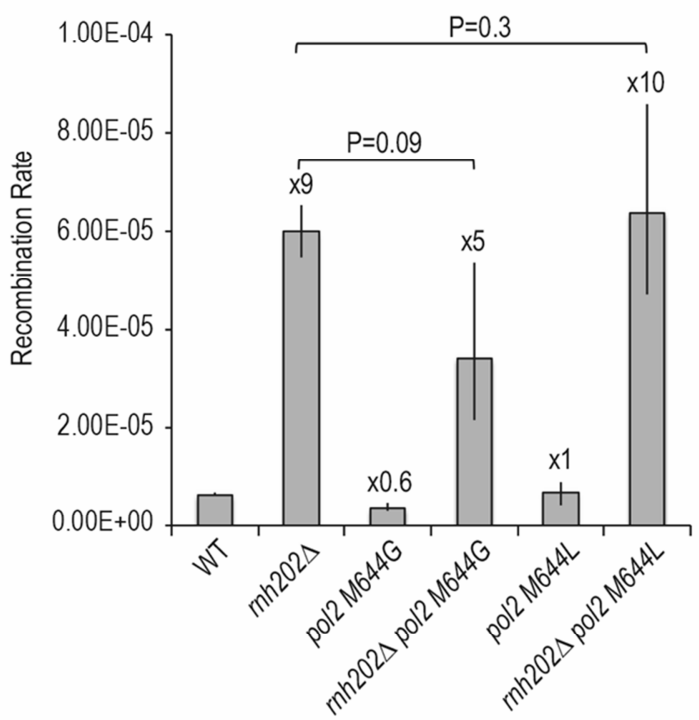

We were concerned that the POL2 mutants could affect the growth rate of cells, particularly in $S$ phase, and that this by itself could provide more opportunity for DNA damage and recombination and hence we determined the doubling times of rnh202 POL2, rnh202 pol2-M644G and rnh202 pol2-M644L strains. We found that the presence of the pol2-M644L mutation did not alter the growth rate of
C

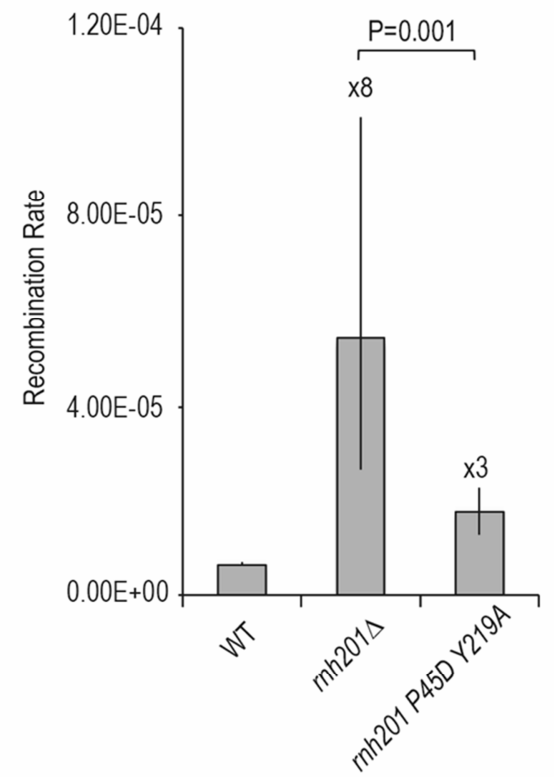

D

\section{B}

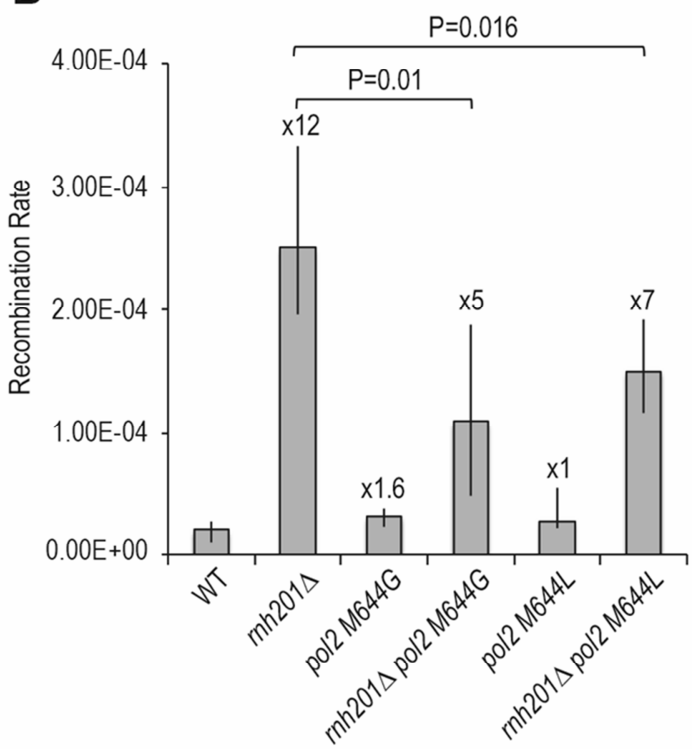

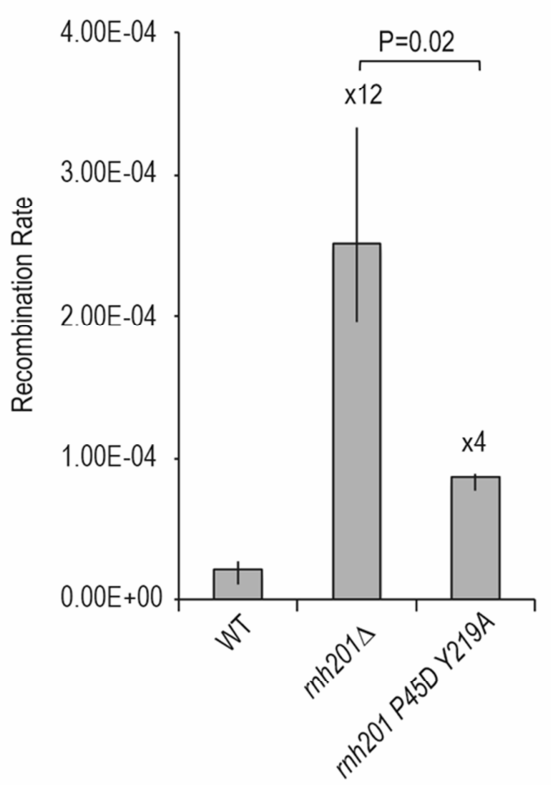

FIGURE 3: Effect of DNA polymerase epsilon mutants and RNase $\mathrm{H} 2$ mutants on recombination rates. (A) Recombination rates using the leu2 duplication reporter in strains with increased (pol2 M644G) or decreased (po/2 M644L) rNMP incorporation are shown. Median rates with $95 \%$ confidence limits $(n=18)$ are presented. (B) Recombination rates using the ade2 duplication reporter in strains with increased (pol2 M644G) or decreased (pol2 M644L) rNMP incorporation are shown. Median rates with 95\% confidence limits $(n=18)$ are presented. (C) Recombination rates in an rnh201 P45D Y 219A strain are shown using the leu2 duplication reporter are shown. Median rates with $95 \%$ confidence limits $(n=18)$ are presented. (D) Recombination rates in an rnh201 P45D Y 219A strain using the ade2 duplication reporter are shown. Median rates with $95 \%$ confidence limits $(n=18)$ are presented. The wild type $(\mathrm{WT})$ and $r n h 201 \Delta$ rates are taken from panel B. 
rnh202A cells, further suggesting that the presence of misincorporated rNMP residues on the leading strand does not account for the increased recombination phenotype. The rnh202 pol2-M644G had a slightly reduced growth rate but this did not account for unaltered recombination rate.

\section{Separation of function rnh201 mutant}

Eukaryotic RNase $\mathrm{H} 2$ enzymes, including the yeast RNase $\mathrm{H} 2$ heterotrimer, can cleave at single rNMPs in DNA but also can cleave at multiple consecutive rNMPs, recognizing the RpR transition bound to DNA [3]. The RpR/DNA structure is termed an R-loop [3]. Using structural information from bacterial RNase $\mathrm{H}$ enzymes with different specificities against RpR/DNA and RpD/DNA hybrids, a separation of function mutant of $R N H 201$, the catalytic subunit of yeast RNase $\mathrm{H} 2$ was constructed [3]. This mutant is completely defective in processing RpD/DNA hybrids, the result of single rNMP residues in DNA, but is able to process RpR/DNA hybrids or R-loops with reasonable efficiency [3]. We used this mutant to assess recombination rates, to determine which substrate stimulated hyperrecombination. We observed that retention of the R-loop processing activity was sufficient to significantly reduce recombination rates (Figure $3 C-D$ ), suggesting that recombination is stimulated by cleavage at RpR/DNA sites, consistent with our results using mutant POL2 alleles that did not greatly affect $r n h 2$ recombination rates.

\section{DISCUSSION}

Hyper-recombination is caused by either creating more recombination-initiating lesions or by changing how a lesion is repaired, from a non-recombination gap filling reaction to a homologous recombination mode [28]. Many hyper-rec mutants fall into the first class, examples being defective components of the DNA replication apparatus, which can leave nicks in DNA that are processed into recombination-initiating breaks. Examples of the second class are mutants that fail to provide anti-recombination activity, such as mutation of the Srs2 DNA helicase such that it fails to removes Rad51 nucleofilaments that thereby prevent recombination.

In the case of mutants in the RNase $\mathrm{H} 2$ complex, these appear to be of the first class in that rNMPs remain in DNA and are ultimately processed by enzymes that form nicks. One possibility for nick formation is through Top1 action, which is known to cleave at rNMPs remaining on the leading strand [20]. As the hyper-recombination phenotype of the rnh2 mutants is dependent on Top1 function, it is logical to infer that Top1 cleaves at such rNMPs on the leading strand and these may ultimately be processed to a double strand break or a single strand gap that can initiate recombination. The study of Conover et al. [25] examined genome instability in diploid yeast, measuring loss of heterozygosity through crossing over and nonallellic homologous recombination. Both of these genome instability events were reduced in a mutant pol2 strain that incorporated fewer rNTPs and increased in a mutant pol2 strain that incorporated more rNTPs. Moreover, the stimulation in genome instability was dependent on Topoisomerase 1. These results are consistent with leading strand rNMPs and subsequent processing by Topoisomerase 1 to create recombinogenic lesions.

In contrast, the study of O'Connell et al. [26], measuring global loss of heterozygosity events in diploid yeast and reciprocal crossing over, did not observe any reduction in instability rate in mutant pol2 strain that incorporated fewer rNTPs. Moreover, the instability rate of an $r n h 201 \Delta$ diploid strain was increased by a $r n h 1 \Delta$ mutation, suggesting that the recombinogenic lesions arise from multiple contiguous rNMP residues or R-loops.

Our results are more in line with those from the O'Connell group, but we have not observed any stimulation of recombination by an $r n h 1 \Delta$ mutation or reduction by over-expression of RNH1. We have examined intrachromatid or intersister chromatid gene conversion, of short gene conversion tracts with a maximum length of 510 nucleotides in haploid strains. The cell cycle timing of recombination and regulation in haploid intrachromosomal recombination may differ from the global diploid loss of heterozygosity studies.

The separation of function rnh201 mutant has provided insight into the origin of recombination by rNMPs. The mutant is completely deficient in the removal of single rNMP residues incorporated into DNA during DNA replication [3]. However, it retains in vitro activity against a tract of six rNMP residues in a RNA:DNA hybrid, suggesting that it can cleave at RpR/DNA sites but not at RpD/DNA sites. Although the in vitro activity of the mutant RNase $\mathrm{H} 2$ is reduced from wild type [3], it is sufficient for in vivo rescue of sgs $1 \Delta r n h 201 \Delta$ synthetic growth defect. We have found that it is sufficient to suppress most of the hyperrecombination seen in an rnh20 2 mutant. The residual increased recombination could arise from a partial loss of activity against RpR/DNA sites in vivo, as suggested by the partial in vitro activity of the mutant [3], or it could reflect that some intrachromosomal gene conversion events are stimulated by single rNMPs in DNA, but they represent a fraction of total events that cannot be detected as altered by the pol 2 mutants. The in vivo origin of the substrate recognized by this rnh201 allele is not clear, but could result from transcription replication conflicts at the replication fork, producing R-loops that eventually result in a recombination lesion in the absence of RNase $\mathrm{H} 2$. However, recombination levels are not further augmented by loss of RNase $\mathrm{H} 1$ in the recombination reporters we have used, and overexpression of RNase $\mathrm{H} 1$ does not reduce recombination levels in an rnh201D mutant. These observations suggest that the RNA:DNA hybrid that stimulates recombination is not a conventional R-loop. Indeed, the recombination-initiating lesion might not be an R-loop arising from transcription, but could instead come from ribonucleotides in DNA that remain in the lagging strand.

As further evidence of the complexity of genome destabilization from embedded rNMPs, we found that chromosome fragment loss was elevated by loss of RNase $\mathrm{H} 2$, but it appears that the action of RNase $\mathrm{H} 1$ on the ensuing lesions causes the chromosome fragment loss. 
We suggest that recombination arises from breaks at the replication fork that occasionally arise during replication. These may come from replication transcription collisions or from consequences of stalled replication. Alternatively, rNMPs on the lagging strand may cause stalling of the replication machinery in the next replication cycle, leading to a double strand break that can initiate recombination. Experiments to address the nature of the RNA:DNA intermediate formed in the absence of RNase $\mathrm{H} 2$ activity is currently under investigation.

\section{MATERIALS AND METHODS \\ Strains}

All strains used in this study are listed in Table S1.

\section{Recombination and chromosome loss rates}

Recombination rates were performed using the leu2ecoRI::URA3::leu2-bstEIl system as described [24] or the ade2n::TRP1::ade2-1-Sce1 as described [29]. Strains were streaked on YPD medium or selective medium to obtain single colonies. At least 12 or 18 independent cultures with a minimum of two isolates per genotype were used to determine rates and $95 \%$ confidence limits [30]. The transcription recombination reporter gal10-kpnl::URA3::gal10-3' $\Delta$ was used as described [15] Chromosome fragment loss was determined as described [31], using at least three independent strains for each genotype. Recombination rates with Rnh1 over-expression was performed using ade2-n::TRP1::ade2-1-Sce1 strains transformed with pRS416-GAL-RNH1(URA3). At least two strains of each genotype with the plasmid were streaked to SC-URA glucose medium. After growth, 12 independent colonies were used to inoculate liquid cultures of SC-URA glucose medium and 12 independent colonies were used to inoculate liquid cultures of

\section{REFERENCES}

1. Nick McElhinny SA, Watts BE, Kumar D, Watt DL, Lundstrom EB, Burgers PM, Johansson E, Chabes A, Kunkel TA (2010). Abundant ribonucleotide incorporation into DNA by yeast replicative polymerases. Proc Natl Acad Sci U S A 107(11): 4949-4954.

2. Cerritelli SM, Crouch RJ (2009). Ribonuclease $H$ : the enzymes in eukaryotes. FEBS J 276(6): 1494-1505.

3. Chon H, Sparks JL, Rychlik M, Nowotny M, Burgers PM, Crouch RJ, Cerritelli SM (2013). RNase $\mathrm{H} 2$ roles in genome integrity revealed by unlinking its activities. Nucleic Acids Res 41(5): 3130-3143.

4. Sparks JL, Chon H, Cerritelli SM, Kunkel TA, Johansson E, Crouch RJ, Burgers PM (2012). RNase $\mathrm{H} 2$-initiated ribonucleotide excision repair. Mol Cell 47(6): 980-986.

5. Aguilera A, Garcia-Muse T (2012). R loops: from transcription byproducts to threats to genome stability. Mol Cell 46(2): 115-124.

6. Gan W, Guan Z, Liu J, Gui T, Shen K, Manley JL, Li X (2011). R-loopmediated genomic instability is caused by impairment of replication fork progression. Genes Dev 25(19): 2041-2056.

7. Hamperl S, Cimprich KA (2014). The contribution of cotranscriptional RNA:DNA hybrid structures to DNA damage and genome instability. DNA Repair (Amst) 19:84-94.

8. Kim N, Jinks-Robertson S (2012). Transcription as a source of genome instability. Nat Rev Genet 13(3): 204-214.
SC-URA galactose medium. After growth for 24 hours, cells were collected by centrifugation, washed, resuspended in $1 \mathrm{ml}$ water, and used at appropriate dilutions for fluctuation test analyses.

\section{ACKNOWLEDGEMENTS}

This work was supported by National Institutes of Health grants R01GM053738 and R01CA146940. We thank Susana Cerritelli and Lorraine Symington for strains and plasmids.

\section{SUPPLEMENTAL MATERIAL}

All supplemental data for this article are available online at www.microbialcell.com.

\section{CONFLICT OF INTEREST}

The authors declare no conflict of interest.

\section{COPYRIGHT}

(C) 2016 Epshtein et al. This is an open-access article released under the terms of the Creative Commons Attribution (CC BY) license, which allows the unrestricted use, distribution, and reproduction in any medium, provided the original author and source are acknowledged.

Please cite this article as: Anastasiya Epshtein, Catherine J. Potenski, and Hannah L. Klein (2016). Increased spontaneous recombination in RNase $\mathrm{H} 2$-deficient cells arises from multiple contiguous rNMPs and not from single rNMP residues incorporated by DNA polymerase epsilon. Microbial Cell 3(6): 248-254. doi: 10.15698/mic2016.06.506

9. Aguilera A (2005). mRNA processing and genomic instability. Nat Struct Mol Biol 12(9): 737-738.

10. Wahba L, Amon JD, Koshland D, Vuica-Ross M (2011). RNase $H$ and multiple RNA biogenesis factors cooperate to prevent RNA:DNA hybrids from generating genome instability. Mol Cell 44(6): 978-988.

11. Chan YA, Aristizabal MJ, Lu PY, Luo Z, Hamza A, Kobor MS, Stirling PC, Hieter $P$ (2014). Genome-wide profiling of yeast DNA:RNA hybrid prone sites with DRIP-chip. PLoS Genet 10(4): e1004288.

12. El Hage A, Webb S, Kerr A, Tollervey D (2014). Genome-wide distribution of RNA-DNA hybrids identifies RNase $H$ targets in tRNA genes, retrotransposons and mitochondria. PLoS Genet 10(10): e1004716.

13. Saxe D, Datta A, Jinks-Robertson S (2000). Stimulation of mitotic recombination events by high levels of RNA polymerase II transcription in yeast. Mol Cell Biol 20(15): 5404-5414.

14. Sikdar N, Banerjee S, Zhang H, Smith S, Myung K (2008). Spt2p defines a new transcription-dependent gross chromosomal rearrangement pathway. PLoS Genet 4(12): e1000290.

15. Thomas BJ, Rothstein R (1989). Elevated recombination rates in transcriptionally active DNA. Cell 56(4): 619-630. 
16. Castellano-Pozo M, Garcia-Muse T, Aguilera A (2012). R-loops cause replication impairment and genome instability during meiosis. EMBO Rep 13(10): 923-929.

17. Stuckey R, Garcia-Rodriguez N, Aguilera A, Wellinger RE (2015). Role for RNA:DNA hybrids in origin-independent replication priming in a eukaryotic system. Proc Natl Acad Sci U S A 112(18): 5779-5784.

18. Nick McElhinny $S A$, Kumar $D$, Clark $A B$, Watt $D L$, Watts $B E$, Lundstrom EB, Johansson E, Chabes A, Kunkel TA (2010). Genome instability due to ribonucleotide incorporation into DNA. Nat Chem Biol 6(10): 774-781.

19. Kim N, Huang SN, Williams JS, Li YC, Clark AB, Cho JE, Kunkel TA, Pommier $Y$, Jinks-Robertson $S$ (2011). Mutagenic processing of ribonucleotides in DNA by yeast topoisomerase I. Science 332(6037): 1561-1564.

20. Williams JS, Clausen AR, Lujan SA, Marjavaara L, Clark AB, Burgers PM, Chabes A, Kunkel TA (2015). Evidence that processing of ribonucleotides in DNA by topoisomerase 1 is leading-strand specific. Nat Struct Mol Biol 22(4): 291-297.

21. Williams JS, Smith DJ, Marjavaara L, Lujan SA, Chabes A, Kunkel TA (2013). Topoisomerase 1-mediated removal of ribonucleotides from nascent leading-strand DNA. Mol Cell 49(5): 1010-1015.

22. Potenski CJ, Niu $H$, Sung $P$, Klein $H L$ (2014). Avoidance of ribonucleotide-induced mutations by RNase $\mathrm{H} 2$ and Srs2-Exo1 mechanisms. Nature 511(7508): 251-254.

23. Reijns MA, Rabe B, Rigby RE, Mill P, Astell KR, Lettice LA, Boyle $S$, Leitch A, Keighren M, Kilanowski F, Devenney PS, Sexton D, Grimes G, Holt IJ, Hill RE, Taylor MS, Lawson KA, Dorin JR, Jackson AP (2012). Enzymatic removal of ribonucleotides from DNA is essential for mammalian genome integrity and development. Cell 149(5): 10081022.
24. Aguilera A, Klein HL (1988). Genetic control of intrachromosomal recombination in Saccharomyces cerevisiae. I. Isolation and genetic characterization of hyper-recombination mutations. Genetics 119(4): 779-790.

25. Conover HN, Lujan SA, Chapman MJ, Cornelio DA, Sharif R, Williams JS, Clark AB, Camilo F, Kunkel TA, Argueso JL (2015) Stimulation of Chromosomal Rearrangements by Ribonucleotides. Genetics 201(3): 951-961.

26. O'Connell K, Jinks-Robertson S, Petes TD (2015). Elevated Genome-Wide Instability in Yeast Mutants Lacking RNase H Activity. Genetics 201(3): 963-975.

27. Cho JE, Kim N, Li YC, Jinks-Robertson S (2013). Two distinct mechanisms of Topoisomerase 1-dependent mutagenesis in yeast. DNA Repair (Amst) 12(3): 205-211.

28. San Filippo J, Sung $P$, Klein H (2008). Mechanism of eukaryotic homologous recombination. Annu Rev Biochem 77:229-257.

29. Mozlin AM, Fung CW, Symington LS (2008). Role of the Saccharomyces cerevisiae Rad51 paralogs in sister chromatid recombination. Genetics 178(1): 113-126.

30. Spell RM, Jinks-Robertson S (2004). Determination of mitotic recombination rates by fluctuation analysis in Saccharomyces cerevisiae. Methods Mol Biol 262:3-12.

31. Marrero VA, Symington LS (2010). Extensive DNA end processing by exo1 and sgs1 inhibits break-induced replication. PLoS Genet 6(7): e1001007. 